\title{
Zur Wirkung der Balsamica.
}

\author{
Von \\ Doz. Dr. Rudolf Winternitz, \\ Leiter der Hautabteilung an der dentschen Universitïtspoliklinik.
}

Bei einer experimentellen Arbeit "Über die entziindungswidrige Wirkung ätherischer Öle"1) zur Erklärung der Heilwirkung, welche Balsamica bei blennorrhoischen Entzündungen der Harnwege entfalten können, habe ich gesehen, daß diese Stoffe auch toxische Wirkungen haben.

Wie P. Meißner, $\left.{ }^{2}\right)$ der über Versuche mit Ol. Santali und Gonorol im Vorjahr berichtete, hatte ich gefunden, daß eine beträchtliche Zahl von Tieren (Kaninchen) nach Verfütterung von einigen Grammen ( $10 \mathrm{~g}$ oder darüber) von Ol. Santali oder Bals. Copaivae, welche in Tagesdosen von $1 / 2-2 g$ erfolgte, noch während der Versuche oder nach einiger Zeit zu Grunde ging.

Da nach der herkömmlichen Anschauung Terpene und terpenhaltige Stoffe intensive Wirkungen auf die Nieren hervorrufen, so suchte ich die Todesursache in einer Schädigung der Nieren, konnte aber bei den Tieren bei Santal- und Copaivafütterung während der Versuchszeit nur eine Steigerung der Diurese und eine nicht einmal konstant auftretende geringe Eiweißausscheidung bei Fehlen von morphotischen Elementen der Niere finden. (Siehe auch P. Meißner.)

Auch die Untersuchung der Nieren nach dem Tode oder der Tötung der Tiere zeigte im wesentlichen normale Verhältnisse. Es fiel

1) Arch. f. exper. Path. und Pharmakol. Bd. XLVI.

\$) Dermatol. Zentralbl. 1901. Nr. 10. 
bloß eine etwas stärkere Gelbfärbung der Rindenpartien der Nieren auf, doch zeigten die von der Niere frisch angefertigten mikroskopischen Präparate keine Verfettung. Weiters wurden einzelne Blutungen in den Rinden- oder Markpartien gefunden. Die Nierenkanälchen und ihre Epithelien waren normal. Auch die übrigen Sektionsbefunde klärten über die Todesursache nicht auf.

Die Tiere waren sehr abgemagert, ihr Fell struppig, ihr Unterhautgewebe und ihre Muskulatur sehr trocken, in den Lungen manchmal begrenzte pneumonische Herde. Im Magen ältere, nach den Balsamicis stark riechende Speisereste, der Darm leer, in seiner Substanz verdünnt, die Darmserose anscheinend stärker venös injiziert.

Die Unklarheit dieses Befundes veranlaßte mich noch, einige Versuche mit 0l. Santal. und Bals. Copaivae bei anderen Tieren vorzunehmen und verwendete ich hiezu kleine Frösche, weiße Mäuse und Ratten; weiters versuchte ich bei diesen Tieren und Kaninchen noch andere in den letzten Jahren empfohlene Balsamica, so das von Riehl') in die Therapie eingeführte Gonorol, das von $\mathrm{Ge} \mathrm{m}$ und $\mathrm{Hu}$ ertas ${ }^{2}$ ) empfohlene Ol. cedri atlanticae. (sowie den Gurjunbalsam). ${ }^{3}$ ) Ich beobachtete, daß kleine Frösche nach Injektion von $1 / 1-1 / 2 \mathrm{~cm}^{3}$ ol. Santal oder Gonorol in den Lymphsack nach $1-3$ Stunden, weiße Mäuse nach subkutaner Injektion ron $0.3 \mathrm{~cm}^{3}$ Santal, beziehentlich $0.25-0.5 \mathrm{~cm}^{3}$ Gonorol innerhalb oder nach 9 Stunden zu grunde gingen. Eine größere weiße Ratte wurde nach subkutaner Injektion von $0.9 \mathrm{~cm}^{3}$ Santalöl sehr elend, erholte sich aber nach 2 Tagen wieder und wurde außer Versuch gestellt. Kaninchen verendeten nach Verfütterung von Gonorol und 01. cedri atlant. in ungefähr derselben Zeit und unter denselben Symptomen, wie nach 0l. Santali und Bals. Copaivae.

Die Analyse der Vergiftungserscheinungen zeigte bei dem Kaltblüter (Frosch) eine kurze Phase leichter Unruhe und eine längere dauernde Periode der Trägheit mit allmählich abnehmender Reflexerregbarkeit. Während dieser zweiten Phase wurden die Atembewegungen seltener, oberflächlicher und sistierten zeitweise durch halbe Minuten und noch länger. Das bloßgelegte Herz zeigte stetige Abnahme der Zahl und Intensität der Kontraktionen. ${ }^{4}$ ) Die Reaktion der Muskeln aber, vom

1) Wiener klin. Wochenschr. 1898. Nr. 52.

2) Bericht von Schimmel \& C o. April 1902. pag. 11.

3) Kunkel: Handbuch der Toxikologie. 1901. pag. 971.

4) Versuch: kleiner Frosch:

6 Uhr 10 Min. Herz bloßigelegt; 
Ischiadicus und rom Muskel direkt geprüft, blieb sowohl während des Lebens der Tiere, als kurz nach dem Tode unversehrt. Die Todesursache ist also in einer die Centra der Herzbewegung und der Atmung betreffenden Lähmung gelegen.

Bezüglich der Todesursache des Warmblüters (Kaninchen) konnte ich nichts sicheres eruieren. Da die Freßlust der Tiere während der Fütterung mit Balsamicis auffallend zurückging und gänzlich anfhörte, das Körpergewicht stetig sank ${ }^{1}$ ) und um $1 / 3$ und mehr herabging, auch die sonstigen Erscheinungen bei der Sektion keinen Schluß nach einer bestimmten Richtung gestatteten, so habe ich auf die Inanition, als eine wesentliche wichtige Ursache des Todes rekurriert.

Einigemal, aber nicht konstant, fand ich, daß die eiweißlösende Kraft des Magensekrets") schwächer war, als die des normalen Kontrollmagens, auch das Darmsekret zweier Tiere ${ }^{3}$ ) erwies sich schwächer diastatisch wirkend (nicht konstant).

In zwei mit den entsprechenden Kautelen, d. i. einer entsprechenden Hunger- und Fütterungsperiode durchgeführten Stoffwechselversuchen fand ich die Gesamtstickstoffausscheilung im Harn (Kjeldahl) bei den Santaltieren etwas größer als bei den Kontrolltieren, resp. größer als in der der Santalfütterung vorangehenden Periode. Doch entsprach diese Stickstoffsteigerung ziemlich gut der Steigerung der Harnmenge, welche bei den Balsamtieren vorhanden ist.

in 30 Sekunden 27 Pulse;

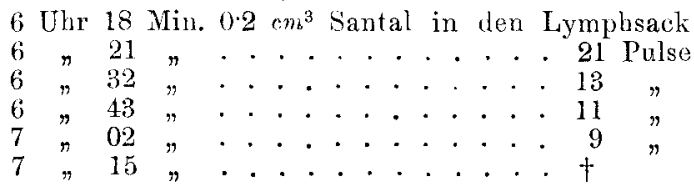

1) Bei Verfütterung von Gurjunbalsnm, welche ich heuer vornahm, an Kaninchen und Hunden beobachtete ich Auftreten von Harzsäuren und Copaivarot, sowie geringen Eiweilimengen, resp. -Spuren im Harı. Die Harnmenge war vermehrt; die Kaninchen nahmen erheblich an Gewicht ab.

2) Eiweißwürfel wurden in schwach salzsaurer Lösung bei Körpertemperatur mit dem filtrierten, salzsauren Fxtrakt der Magenschleimhant durch 1-2 Tage verdaut.

3) Stärkemebllösung, die vorher auf Reduktion geprüft worden, wurde der Einwirkung eines schwach alkalischen (kohlensaures Natron Fxtraktes der Darmschleimhaut bei Körpertemperatur ausgesetzt. 
Es kann somit vurläufig nur die Inanition, deren Einwirkung durch den einigermaßen gesteigerten Stoffzerfall (Diurese, Vermehrung der Stickstoffausscheidung) von tieferer Schädigung sein dürfte, zur Erklärung des Todes der Versuchstiere herangezogen werden.

Über die Erklärung der Heilwirkung der Balsamica ist die herrschende Anschaung weder einheitlich noch genügend gestützt. Sie betont namentlich zwei Wirkungsweisen der Balsamica: die antiseptische und diuretische Wirksamkeit. Die erstere steht nur bei direktem Kontakt gewisser Stoffe dieser Gruppe, wie Ol. terebintinae, mit tierischen Geweben außer Zweifel; nicht sicher ist es jedoch, ob auch die anderen, namentlich aber ihre Ausscheidungsprodukte in den Harnwegen eine antiseptische Wirksamkeit entfalten.

Valentine hat bei Posner gefunden, daß die Harne von Patienten, welche 0l. Santali genommen hatten, einen guten Nährboden für Gonokokken und andere in der Harnröhre vorkommenden Mikroorganismen abgeben. Ich habe mich in Versuchen, die ich vor Jahren (1889) auf der Klinik des Geheimrat $\mathrm{N}$ eisser angestellt habe, davon überzeugt, daß die Harne von Patienten, welche Ol. Terebint, Ol. Santal., Bals. Copaiv, eingenommen hatten, viel später faulten als Normalharne - ejne schon früher für den Copaivabalsam gemachte Beobachtung. Doch zeigten Züchtungsversuche, bei welchen ich den Copaivaharn zusammen mit Gelatine oder Agar als Nährboden benützte, daß sowohl die Harnbakterien, als andere in die Nährstoffe gebrachten Mikroorganismen aufgingen, wenn auch anfangs langsamer, als in den gleichzeitig beschickten Kontrollharnen.

0 . $\mathrm{Sachs}^{1}$ ) hat im vorigen Jahre bei einer Untersuchung über Harnantiseptica den antiseptischen Wert der genannten Balsamica nicht groß befunden; Urotropin, aber auch schon Salol sind ihnen in dieser Richtung weit überlegen.

Diese Erfahrungen stellten für weitere Versuche bezüglich antiseptischer Wirksamkeit der Balsamica keine günstigen Erfolge in Aussicht.

Sicher günstig für den Ablauf von eitrigen Entzündungen der Harnwege ist dagegen die diuretische Wirksamkeit der

1) Wiener klin. Wochenschr. 1902. Nr. 17 und 18. 
Balsamica. Letztere steht für Ol. terebintinae außer Zweifel; auch für Copaiva wurde sie beobachtet. ${ }^{1}$ )

Bei Ol. Santal, Gonorol, Ol. cedri atlantici (Copaiva) habe jch sie für Dosen von $1 / 2$ bis $1 \mathrm{~cm}^{3}$ bei Kaninchen und Hunden beobachtet. Die Harnmenge kann bei den Tieren auf das doppelte und darüber steigen, sie ist auch bei Entziehung des Wassers bedeutend größer als bei dem dürstenden Kontrolltier.") Der Wert dieser Harnvermehrung für die Heilwirkung an der erkrankten Schleimhaut der Harnwege ist einleuchtend. Die Schleimhaut wird hiedurch häufiger von den auflagernden Sekretmassen und den (Reizstoffe absondernden) Mikroorganismen befreit. Diese Wirkung wird nur dann unerwünscht sein und zu einer Sistierung der Balsamverabreichung führen, wenn ein bedeutender Entzündungsgrad oder die heftige Entzündung gewisser Regionen der Schleimhaut (urethra posterior) eine Ruhigstellung der Schleimhaut und demnach eine Verringerung der Diurese erheischen.

Allem Anscheine nach ist nun auch diese dinretische Wirkung der Balsamica allein für sich nicht im stande, ihre Heilwirkung zu erklüren; denn von anderen diuretisch wirkenden Mitteln, wie z. B. Jodkali, ist eine gleich günstige Einwirkung auf Blennorrhoen nicht bekannt. Ich unternahm es deshalb, zu untersuchen, ob sich ein direkter und meßbarer Einfluß bei der Verabreichung von Balsamicis (Copaiva, Santal, Ol. terebintin.) am Orte einer Entzündung $\mathrm{g}^{3}$ ) nachweisen lasse.

Die Untersuchung zerfällt naturgemäls in zwei. Es kounte geprift werden, ob der Grad oder der Ablauf der Entzündung irgend eine Änderung erfahren. Diese Fragen experimentell zu beantworten, setzte ich bei Tieren kntziindungen an einer Schleimhaut, resp. einem endoteltragenden Organ. Hiezu benitzte ich chemisch wirkende Reizstoffe, um nicht durch Einführung von Bakterien einem Wechsel in der Jenge der Reizstoffe (welche von den Bakterien ins Gewebe gelangen) ausgesetzt zu sein.

1) Quincke: Arch. f. exper. Path. und Pharm. XVII. 277.

2) 1. Fin Santaltier entleert in drei Tagen zusammen $150 \mathrm{~cm}^{3} \mathrm{Harn}$ (3., 4., 5. Versuchstag); das Kontrolltier entlerrt in drei Tagen zusammen $60 \mathrm{~cm}^{8}$ Harn (3., 4., 5. Versuchstag). - 2. Ein Copaivtier entleert in zwei Tagen zusammen $233 \mathrm{~cm}^{3}$ Harn (4. und 5. Versuchstag); das Kontrolltier. entleert in zwei Tagen zusammen $79 \mathrm{~cm}^{3}$ Harn (4. und 5. Versuchstag).

s) Vergl. M. M a y er, Münch. m. W. 1902. Nr. 32. 
Die erste Frage mußte negativ oder fast negativ beantwortet werden. Denn nur bei Anwendung der geringsten Menge. eines von mir versuchten reizenden Stoffes, der an der Conjunctiva Entzündung hervorruft, konnte ich, und zwar nicht einmal konstant, einen kleinen Einfluß beobachten, indem bei dem mit Santal vorbehandelten Tier eine unbedeutende Abmilderung der Chemosis und Exsudation sichtbar war. Dagegen war die Untersuchungsreihe bezüglich des Einflusses auf den Ablauf der Entzündung bei der größeren Reihe der Versuche der Erklärung günstiger.

Ich setzte bei Hunden und Kaninchen, von welchen ein Teil die Balsamica verfüttert bekam, eitrige Pleuritiden, indem ich den Tieren unter aseptischen Kautelen kleine Mengen eines sterilisierten Aleuronatgemisches (Wasser) in die Pleurahöhle einspritzte.

Nach 1-3 Tagen wurden die Tiere getötet, die Pleuraböhlen geöffnet und die Menge des Exsudats (sowie seine Beschaffenheit, Trockenrückstand) bestimmt. Hiebei zeigte sich, daß in der größeren Mehrzahl der Versuche die Exsudatmenge bei den mit Balsamicis gefütterten Tieren kleiner war, als bei den Kontrolltieren.

Gewiß sind diese Versuche mit dem Fehler behaftet, daß die Menge des Aleuronats, welche eingespritzt wurde, nur annähernd bestimmbar war, und weiters, daß sich namentlich bei der Einspritzung durch das Zusammenballen das Aleuronatpulver, trotz seiner Verreibung, Schwierigkeiten einstellten.

Dieser letztere Versuchsmangel wurde heuer, wo ich Gonorol auf seine Wirksamkeit prüfte, dadurch beseitigt, daß ich die Injektionsspritze wagrecht hielt, wodurch eine Verstopfung der Kanüle vermieden wurde. Es fielen auch wahrscheinlich demzufolge die Unterschiede bei den Versuchen nicht so groß aus; indessen ist doch auch bei den neuen Versuchen die Exsudatmenge der Balsamtiere etwas kleiner gefunden worden, als bei den Kontrolltieren.

Beifolgend einige Belege:

Balsam. Copaiv. 5tägige Fütterung mit Copaiva, zusammen $4^{1} / 2 \mathrm{~cm}^{3}, 24$ Stunden nach der Injektion von je $6 \mathrm{~cm}^{3}$ Aleuronat bei Versuchs- und Kontrolltier werden beide getötet. Das Versuchstier hat ungefähr $1 / 4 \mathrm{~cm}^{3}$, das Kontrolltier $4^{1 / 2} \mathrm{~cm}^{3}$ Exsudat im Pleurasack.

Ol. Cubebarum. 6tägige Fütterung mit Ol. Cubeb.; zusammen 70 Tropfen. $1^{1 / 2}$ Tage nach der Aleuronatinjektion bei allen Tieren (2 Versuchs- und 2 Kontrolltieren) werden dieselben getötet. Die zwei Versuchstiere haben $4 \frac{1}{2}$ und $4 \mathrm{~cm}^{3}$ Exsudat; die zwei Kontrolltiere haben $16^{3} / \mathrm{cm}^{3}$ Exsudat. 
Ol. Santal. 3tägige Fütterung mit Ol. Santal., zusammen $3^{3 / 4} \mathrm{~cm}^{3}$ Ol. Sant. 2 Tage nach der Aleuronatinjektion getötet. 'Das Versuchstier (Hund) hat $15 \mathrm{~cm}^{3}$ Exsudat; das Kontrolltier (Hund) hat $31 \mathrm{~cm}^{3}$ Exsudat. Gonorol (heuer gemachte Versuche): Resultat.

1. Versuch mit 4 Thieren (2 Versuchs- and 2 Kontrolltiere) ohne

2. Versuch mit 4 Tieren (2 Versuchs-, 2 Kontrolltieren). Jedes Versuchstier bekommt innerhalb 5 Tagen im ganzen $2 g$ Gonorol; $1^{1 / 2}$ Tage nach der Aleuronatinjektion werden die Tiere getötet. Die Versuchstiere haben $13 \mathrm{~cm}^{3}$ und $11 \mathrm{~m}^{3}$ Exsudat; die Kontrolltiere haben $24 \mathrm{~cm}^{3}$ und $11 \mathrm{~cm}^{3}$ Exsudat.

3. Versuch. Fütterung mit Gonorol durch 6 Tage; zusammen $\tilde{5}^{1 / 4} \mathrm{~cm}^{\mathbf{3}}$ Gonorol. Tötung der Tiere 2 Tage nach der Aleuronatinjektion. Das Versuchstier hat $2 \mathrm{~cm}^{3}$ Exsudat; das Kontrolltier hat $6 \mathrm{~cm}^{3}$ Exsudat.

Ol. terebinth. pur. (4 Tiere.) Die Versuchstiere bekommen je 70 Tropfen innerhalb 6 Tagen. Tötung der Tiere 2 Tage nach der Aleuronatinjektion. D1e Versuchstiere haben $7 \mathrm{~cm}^{3}$ und $3 \mathrm{~cm}^{3}$ Exsudat; die Kontrolltiere haben $16^{3} / 4$ und $61 / 2 \mathrm{~cm}^{3}$ Exsudat.

Terpin hydrat $(2: 100) 4$ Tiere. Die Versuchstiere bekommen je 1.2 $g$ Terpinbydrat innerhalb 3 Tagen; Tötung der Tiere 2 Tage nach der Aleuronatinjektion. Die Versuchstiere haben $12 \mathrm{~cm}^{3}$ und $3 \mathrm{cn}^{3}$ Exsudat; die Kontrolltiere haben $8 \mathrm{em}^{3}$ und $23 \mathrm{en} \iota^{3}$ Exsudat.

Diese Versuche sind einer größeren Anzahl gleichsinniger als Beispiel entnommen. In einer geringeren Anzahl von Versuchen sind Unterschiede in der Exsudatmenge bei den Versuchstjeren nicht zu verzeichnen gewesen.

Was die qualitative Beschaffenheit der Kxsudate bei den beiden Reihen von Tieren, den Versuchs- und Kontrolltieren betrifft, so schien es in einigen Versuchen, daß das Exsudat der Balsamtiere trüber und dicker sei, so daß man hätte annehmen können, daß bei ihnen eine stärkere Resorption der flüssigen Bestandteile des Exsudats stattgefunnen habe. Da bei den Balsamtieren gleichzeitig - wie schon erwähnt worden die Diurese gesteigert ist, so hätte sich die raschere Verminderung des Exsudats sehr einfach durch die reichliche Harnabsonderung erklären lassen.

Aber es zeigte sich einerseits bei der Trockenbestimmung des Exsudats, da $\$$ bei Balsam- und Kontrolltieren kein Unterschied vorhanden sei, also bei den ersteren eine raschere Resorption sowohl der zelligen als der flüssigen Exsudatbestandteile erfolge, andererseits lehrten Versuche, daß die Steigerung der Diurese durch andere Stoffe (Jodkali, Harn- 
stoff) eine Verminderung des Aleuronatexsudates nicht so befördern, wie die Verfütternng der Balsamica. Vorderhand fehlt mir eine präzise Erklärung für diese resorptionsfördernde Wirksamkeit der Balsamica und ich habe deshalb eine Hypothese herangezogen.

Die Balsamica gehören zu jenen Stoffen, welchen die Eigenschaft, Leukocyten anzuziehen, zukommt. Wenn diese Stoffe im Blute, welches entzündete Gewebe durchströmt, vorhanden sind, so zoürfte das entzündliche Exsudat rascher resorbiert werden und das Gewebe seine normale Beschaffenheit früher wiedererlangen.

Fasse ich die Ergebnisse meiner Versuche unter Vergleichung mit den Symptomen der klinischen Beobachtung und indem ich sicheres von wahrscheinlichem scheide, zusammen, so folgt: die Balsamica rufen bei kleineren Tieren (Kalt- und Warmblütlern) toxische, beziehungsweise tötliche Wirkungen hervor. Eine sichere Erklärung für den Tod der warmblütigen Tiere konnte ich nicht finden. Die beim Tiere erzielten Giftwirkungen sind in geringerem Grade bei der medikamentösen Verabreichung beim Menschen zu beobachten. So die Magen-, Darmerscheinungen, beim Menschen als Appetitsstörung, zeitweilige Stuhlverstopfung, im Tierexperiment als völliges Aufhören der Freßlust, welches zur tötlichen Inanition führen kann; weiters Erscheinungen von Seite der Nieren $u$. zw. geringe Eiweißausscheidung und Auftreten von etwas Blut im Harn beides beim Menschen und in den mitgeteilten Tierversuchen beobachtet. ')

Beim Menschen durch Genuß von Balsamicis entstehende Exantheme sind im Tierversuche nicht beobachtet worden, wol deshalb, weil nicht geeignete Tiere gewält worden sind, Bezüglich der therapeutischen Wirksamkeit ist die diuretische Wirkung der Balsamica und ihr günstiger Einfluß bei der Therapie zweifellos. Als nicht absolut sicher, aber als wahrscheinlich ergibt sich ein günstiger direkter Einfluß der Balsamica auf den Ablauf der Entzündungserscheinungen, indem das entzündliche Exsudat schneller resorbiert, das Gewebe früher zur Norm zurückgeführt wird.

Diese Versuche sind im Universitätsinstitute fïr Pharmakologie (Prof. Pohl) ausgeführt worden.

1) P. Meisener hat nach Santal mehrfach hämorrhagische Nephritis beim Kaninchen gesehen. 